\title{
Principios de Diseño en las aplicaciones de m-Salud Mental orientadas al control psicosocial
}

\author{
Salvador Prefasi Gomar ${ }^{a}$, Teresa Magal-Royo ${ }^{a}$ \\ ${ }^{a}$ Universitat Politècnica de Valencia, Camino de Vera s/n, Valencia 46022, Spain | tmagal@degi.upv.es
}

\begin{abstract}
Resumen
Las tecnologías digitales tienen el potencial de cambiar el método de control sanitario y la atención médica personalizada en el ámbito de la Salud Mental. Sin embargo, para integrarlas en un sistema de prestación de servicios de salud asistencial, los médicos y/o terapeutas, diseñadores y programadores necesitan conocer los parámetros más importantes que influyen directamente en el uso de aplicaciones interactivas online orientada a pacientes de Salud Mental. Para poder diseñar este tipo de aplicaciones con una base sólida y que proporcione a los usuarios una Experiencia de Usuario (UX) óptima es importante adherirse a una serie de principios relacionados con el diseño para herramientas digitales. Nos referimos a los Principios para el Desarrollo Digital que se crearon para guiar sobre mejores prácticas en el diseño de programas de prestación de servicios compatibles con la tecnología digital.
\end{abstract}

Palabras clave: m-Salud Mental, innovación sanitaria, Experiencia del Usuario (UX), Principios de diseño, Desarrollo Digital, terapias psicosociales.

\section{Introducción}

La revolución tecnológica que se está viviendo abarca todos los aspectos de la sociedad, incluidas por supuesto, la medicina y la salud. A lo largo del siglo XX, se han producido muchos avances tecnológicos en cirugía y en desarrollo de medicamentos. Gracias a Internet y a los dispositivos móviles, la relación entre paciente y médico está cambiando profundamente. La tecnología ha llegado para quedarse, y afrontar nuevos retos, entre los que se encuentra la reestructuración y transformación del sector sanitario, gracias a los avances tecnológicos que 
contribuyen a desarrollar y a mejorar la eficiencia sanitaria desde un punto de vista humano y económico.

Todos estos avances en el sector de la salud necesitan de herramientas, aplicaciones y plataformas digitales específicas. Este tipo de herramientas deben ser lo más eficientes y eficaces posibles para alcanzar los objetivos marcados por los profesionales sanitarios, tanto a nivel de hardware como a nivel de software. Si bien es cierto que, en el campo de las discapacidades físicas, ha habido una importante revolución tanto en el aspecto hardware como software, en la discapacidad mental e intelectual, este avance no se ha producido al mismo ritmo. Actualmente, en el caso de las aplicaciones empleadas tanto para la rehabilitación (cognitiva/social), como para el control y evaluación del comportamiento y estado de ánimo del paciente, todas ellas dirigidas a usuarios con problemas mentales, se hace indispensable revisar conceptos, metodologías, tecnologías y procesos adaptados para llegar a un diseño inclusivo para estos colectivos. Así pues, para diseñar aplicaciones con una base sólida que proporcione al usuario una Experiencia de Usuario $(U X)$ óptima es importante adherirse a una serie de principios relacionados con el diseño para herramientas digitales (Waugaman, 2016). Nos referimos a los Principios para el Desarrollo Digital.

\section{La importancia de la intervención del paciente en el diseño de aplicaciones orientadas al control psicosocial}

La evidencia de la viabilidad, la aceptación y la eficacia de las intervenciones basadas en web y en dispositivos móviles en salud mental está creciendo rápidamente, en particular para enfermedades como la ansiedad y la depresión (Arnberg et al., 2014). Estudios preliminares indican que las intervenciones en línea pueden tener una utilidad similar para enfermedades más severas y duraderas, como el Trastorno Bipolar y la psicosis (Hidalgo-Mazzei et al., 2015), (Naslund et al., 2015). Con demasiada frecuencia, en el campo del desarrollo de herramientas y aplicaciones digitales, se diseñan proyectos y sistemas sin la participación suficiente de los interesados, cuyo compromiso y opinión son críticos para el éxito a largo plazo. Así pues, los proyectos diseñados sin la participación del usuario pueden fallar debido a simples problemas de usabilidad en lugar de deberse a un diseño defectuoso. Se sabe que las personas con problemas de salud mental pueden tener dificultades para participar en intervenciones basadas en la web o en dispositivos móviles, debido al grado de enfermedad, a la situación del paciente 
o a la poca usabilidad de las herramientas actuales, ya que los niveles de su uso, probablemente son más bajos que los de la población en general. De ahí, la importancia de contar con ellos, a la hora de diseñar y desarrollar una aplicación que va a formar parte de su tratamiento en el camino hacia su recuperación. Esta colaboración de pacientes con una enfermedad mental puede realizarse a través de métodos de evaluación, bien antes de la distribución de la aplicación Formative Evaluation, muy recomendable ya que así pueden detectarse carencias durante el proceso de desarrollo que se pueden solventar más fácilmente y con un coste menor o bien durante su vida activa Summative Evaluation. puede resultar más complicada y cara, pero puede servir para futuras actualizaciones de la herramienta. La participación de pacientes con una enfermedad mental en el análisis y evaluación de aplicaciones digitales para su tratamiento, mediante métodos de evaluación como la encuesta, arroja información relevante para recopilar datos interesantes a tener en cuenta a la hora de plantear el diseño de nuevas aplicaciones destinadas al tratamiento de pacientes con una enfermedad mental grave y crónica como el Trastorno Bipolar. Una encuesta realizada a 552 participantes con Trastorno Bipolar (Murnane et al., 2016), devuelve interesantes conclusiones sobre la utilización de aplicaciones móviles como herramienta para la rehabilitación de la enfermedad mental y el autocontrol de los pacientes ante síntomas de recaída. Casi dos tercios de los encuestados respondieron que la utilización de aplicaciones para el autocontrol y la rehabilitación del Trastorno Bipolar cambió positivamente su postura frente a la enfermedad, gracias a los beneficios de comportamiento, psicológicos y sociales adoptados por los pacientes. En particular, el seguimiento ayudó a los individuos a:

- Imponer coherencia a su comportamiento afectado por la enfermedad.

- Aumentar la autoconciencia de los patrones del Trastorno Bipolar, los desencadenantes y las estrategias de afrontamiento eficaces.

- Permitir el desarrollo de la autosuficiencia y evitar la autocompasión.

- Facilitar la transmisión de información sobre su estado de ánimo hacia los terapeutas.

Además, los encuestados señalaron que el uso de la tecnología para buscar información y obtener conocimiento sobre el Trastorno Bipolar sin la participación directa del médico o terapeuta, era empoderante y esencial para el manejo efectivo de su condición, ya que les ayudaba a aceptar y comprender mejor su enfermedad. Pero, aunque la tecnología tiene el potencial de aumentar los beneficios mencionados anteriormente, se observa en este estudio que su uso puede tener un efecto de agitación o incluso desencadenar la inestabilidad emocional debido a un diseño inadecuado o a una falta de usabilidad provocando una Experiencia de Usuario pobre. Los participantes también identificaron experiencias problemáticas, surgidas de 
las frustraciones que este tipo de aplicaciones pueden generar en los pacientes, debido a que se aprecian poco intuitivas, no confiables o demasiado genéricas por no estar orientadas al Trastorno Bipolar. Otros problemas que encontraron los encuestados, fue la dificultad para recordar o seguir rutinas de seguimiento, especialmente durante los estados de manía o depresión (Murnane et al., 2016).

Por tanto, es trabajo de los diseñadores de este tipo de aplicaciones, junto a los terapeutas, solucionar estas tensiones a través de un diseño para todos, y teniendo en cuenta una serie de principios y estrategias que nos ayuden a conseguirlo. Por otra parte, Dodd en su estudio User' Experience of an Online Intervention for Bipolar Disorder: Important Lessons for Design and Evaluation, indica una serie de conclusiones muy interesantes, presentadas en forma de factores facilitadores y de barreras, sobre qué características deben tener las aplicaciones online, para web o para dispositivos móviles, y qué aspectos deben evitarse para lograr una buena UX, y así poder integrarlas en su rehabilitación y tratamiento psicosocial (Dodd et al, 2017).

Tabla 1.1 Factores facilitadores y barreras en el uso de herramientas online para la rehabilitación y el tratamiento de pacientes con un Trastorno Bipolar

\begin{tabular}{|c|c|}
\hline FACILITADORES & BARRERAS \\
\hline $\begin{array}{l}\text { La aplicación debe ser simple, directa y con una arquitectura } \\
\text { modular fácil de navegar. Importancia de diseñar una herramienta } \\
\text { simple, usable y accesible para que al usuario le sea fácil y rápido } \\
\text { encontrar la información que busca. }\end{array}$ & $\begin{array}{l}\text { Privacidad en las sesiones. La privacidad es una } \\
\text { preocupación clave sobre los enfoques digitales, } \\
\text { sobre todo a la hora de emplear la aplicación en } \\
\text { público, por el temor a la interrupción o a la } \\
\text { divulgación involuntaria de la información. }\end{array}$ \\
\hline $\begin{array}{l}\text { Layout y estética de la aplicación sencilla y normalizada. } \\
\text { Mantener unos estándares de diseño de páginas online y } \\
\text { aplicaciones móviles, normalizando, y señalizando los elementos } \\
\text { que la conforman para facilitarle la navegación al usuario, sin } \\
\text { tener que apelar a su memoria. }\end{array}$ & $\begin{array}{l}\text { Colores demasiado brillantes en partes de la } \\
\text { aplicación. Algunas aplicaciones permiten la } \\
\text { personalización de la apariencia dentro de unos } \\
\text { parámetros. }\end{array}$ \\
\hline $\begin{array}{l}\text { Contenido textural bien redactado y comprensible. Los contenidos } \\
\text { deben estar bien redactados y estructurados, y su extensión debe } \\
\text { ser la adecuada para evitar abrumar al usuario con información } \\
\text { poco útil en ese momento de la búsqueda. }\end{array}$ & $\begin{array}{l}\text { Falta de un soporte técnico y emocional. Esta } \\
\text { desventaja es sobre todo tenida en cuenta por los } \\
\text { participantes que no dominan el mundo digital, y } \\
\text { para los que el contacto humano es } \\
\text { imprescindible. }\end{array}$ \\
\hline $\begin{array}{l}\text { Inclusión de elementos personalizados e interactivos como vídeos } \\
\text { de personas que comparten su experiencia de vida, gráficos de } \\
\text { vida, gráficos de estados de ánimo, etc. Mediante este tipo de } \\
\text { elementos, el paciente se identifica e interactúa con dicho } \\
\text { material, facilitando la autoconciencia para sentirse menos aislado. }\end{array}$ & $\begin{array}{l}\text { Información simplista e impersonal. El hecho de } \\
\text { no personalizar la información y que además no } \\
\text { sea de calidad, hace que los usuarios no vean la } \\
\text { plataforma como una herramienta seria, y pueden } \\
\text { dejar de confiar en ella. }\end{array}$ \\
\hline
\end{tabular}




\begin{tabular}{|c|c|}
\hline $\begin{array}{l}\text { Estrategias de prevención de recaídas. Los pacientes, con este tipo } \\
\text { de contenido, ven un punto de apoyo donde acudir por si necesitan } \\
\text { ayuda. }\end{array}$ & $\begin{array}{l}\text { Estado del paciente. Los pacientes encuentran } \\
\text { dificultad en emplear este tipo de herramientas } \\
\text { cuando están enfermos, es decir durante los } \\
\text { estados de manía o depresión. }\end{array}$ \\
\hline $\begin{array}{l}\text { Confianza en el equipo investigador de donde proviene la } \\
\text { información. El hecho de que los pacientes sepan quién está detrás } \\
\text { del diseño y desarrollo de este tipo de herramientas genera } \\
\text { confianza en ellos. }\end{array}$ & $\begin{array}{l}\text { Longitud de los test de evaluación. Las medidas } \\
\text { para conocer su estado no deben ser demasiado } \\
\text { largas, evitando así que les ocupen mucho tiempo } \\
\text { contestarlos. }\end{array}$ \\
\hline $\begin{array}{l}\text { Información relevante sobre la enfermedad. Para que los pacientes } \\
\text { lo consideren un sitio de referencia para obtener información } \\
\text { fidedigna sobre su enfermedad. }\end{array}$ & $\begin{array}{l}\text { Frecuencia de monitoreo. El paciente prefiere que } \\
\text { se realice de forma diaria a semanal, para sentirse } \\
\text { más seguro y arropado por el equipo terapéutico. }\end{array}$ \\
\hline $\begin{array}{l}\text { Recordatorios. Importancia de estar en contacto con el equipo de } \\
\text { terapeutas por sentirles más cerca, y sentirse ellos mismos menos } \\
\text { desprotegidos. }\end{array}$ & $\begin{array}{l}\text { Comunicación a través de la propia plataforma. } \\
\text { Los pacientes preferirían recibir la información a } \\
\text { través de la propia plataforma en lugar de } \\
\text { emplear medios alternativos como SMS o correos } \\
\text { electrónicos. }\end{array}$ \\
\hline $\begin{array}{l}\text { Satisfacción personal y mejora la autoestima. Proporcionan pautas } \\
\text { para facilitar que el paciente piense por sí mismo, y lo más } \\
\text { importante, en sí mismo. }\end{array}$ & $\begin{array}{l}\text { Exceso de texto y lenguaje complicado. Cuando } \\
\text { hay un exceso de texto y el lenguaje empleado no } \\
\text { es apropiado, se hace difícil encontrar los } \\
\text { conceptos y entenderlos. }\end{array}$ \\
\hline $\begin{array}{l}\text { Deshacer errores de forma sencilla. En el caso de cometer un } \\
\text { error, debe ser fácil poder volver atrás. }\end{array}$ & $\begin{array}{l}\text { Mal funcionamiento de la aplicación. Resulta } \\
\text { incómodo y desmotivador que la aplicación no } \\
\text { responda o nos devuelva mensajes de error. }\end{array}$ \\
\hline $\begin{array}{l}\text { Materiales complementarios útiles. Facilitar el acceso a materiales } \\
\text { de apoyo para los pacientes, como instrucciones paso a paso } \\
\text { individualizadas, o un breve manual de usuario sobre la propia } \\
\text { aplicación. }\end{array}$ & $\begin{array}{l}\text { Aplicación lenta. Que la aplicación cueste de } \\
\text { cargarse o que sea lenta, a la hora de navegar por } \\
\text { ella. }\end{array}$ \\
\hline $\begin{array}{l}\text { Navegación explicita e hipervínculos inclusivos. Debemos } \\
\text { facilitar la navegación, haciéndola muy intuitiva y hacer muy } \\
\text { visibles los hipervínculos. }\end{array}$ & \\
\hline
\end{tabular}

Fuente: Dodd et al., 2017

\section{El Diseño de aplicaciones sanitarias orientadas al usuario: Principios para el Desarrollo Digital}

A finales de la primera década del año 2000, y ante las dificultades que planteaba la implementación de programas digitales para pacientes con problemas de salud mental, diversas 
organizaciones como la Organización Mundial de la Salud, el Fondo de las Naciones Unidas para la Infancia, la Agencia de los EEUU para el Desarrollo Internacional, la Agencia Sueca para el Desarrollo Internacional y la Fundación Bill y Melinda Gates crearon los Principios para el Desarrollo Digital. (Bauer et al., 2018). Estos principios están diseñados para servir como un enfoque heurístico para considerar cómo se pueden traducir, en la práctica diaria, los éxitos y los fracasos en el desarrollo digital. Estas directrices no resuelven todos los desafíos existentes para el uso justo, efectivo, sostenible y seguro de la tecnología en proyectos de desarrollo en general, y de salud en particular (Waugaman, 2016). Por esta razón, no están diseñados para servir como una lista de verificación. De hecho, sería difícil, sino imposible, implementar todos los principios simultáneamente. A medida que avanzan y se desarrollan las Tecnologías de la Información y la Comunicación (TIC), estos principios deben ir revisándose y actualizándose. En el caso del ámbito sanitario orientado a aplicaciones relacionadas con la Salud Mental, estos principios pueden adaptarse teniendo en cuenta las necesidades, limitaciones y prioridades del paciente con un trastorno mental grave y crónico como el Trastorno Bipolar.

- Diseño con el usuario teniendo en cuenta las limitaciones del paciente y las peculiaridades que éste pueda sufrir durante los diferentes estados de la enfermedad (manía, depresión, eutimia).

- Comprender el ecosistema (comunidad) existente, para conocer las necesidades sociales de la comunidad de personas con problemas de Salud Mental, evitando así la estigmatización.

- Creación de un diseño escalable y por fases que permita al terapeuta obtener resultados generalizables para repetir la experiencia en ecosistemas similares.

- Construir una aplicación interactiva sostenible, que pueda ser mejorada y cambiada en el tiempo, ajustándose a las necesidades, tanto de los pacientes como de terapeutas.

- Impulsarse por los datos. Tomar como referencia los datos obtenidos en los pocos estudios relacionados que existen actualmente en la literatura para este colectivo.

- Uso de datos abiertos, estándares abiertos, código abierto e innovación abierta, para que resulte más sencillo trabajar con este tipo de aplicaciones.

- Reutilizar y mejorar. Analizar las aplicaciones eficaces que ya existen en el mercado y mejorarlas. Este proceso es más económico que empezar un diseño de cero.

- Potenciar la privacidad y seguridad del paciente tanto a nivel personal como de los datos obtenidos. Es importante que el paciente se sienta seguro a la hora de utilizar estas aplicaciones ya que es una de sus principales preocupaciones.

- Ser colaborativo. Crear aplicaciones que permitan fomentar la colaboración entre los agentes sanitarios involucrados en el seguimiento del paciente sin invadir la privacidad del mismo. 
Las aplicaciones que se rigen por estos principios deben crearse a través de un proceso de diseño centrado en el ser humano que debe poner a dos grupos de usuarios finales (pacientes $y$ terapeutas) en el centro de cada etapa del proyecto. En el caso de las aplicaciones sanitarias orientadas a pacientes de Salud Mental, aún es más importante contar con el usuario, porque así se conocen de primera mano las limitaciones que este tipo de pacientes tiene a la hora de enfrentarse, no solo a las nuevas tecnologías, sino también a las debidas a la propia enfermedad. Con su colaboración, el diseño y desarrollo de este tipo de aplicaciones será más rica, ya que desde el principio se dirigirá al público objetivo para la que está destinada, mejorando así la UX de los pacientes.

\section{Conclusiones}

Tanto pacientes como terapeutas coinciden en que las intervenciones online deben ser interactivas, de confianza y estar integradas en un ámbito social más amplio. Se requiere así de una diversidad de enfoques para darle a las personas un rango de opciones y elecciones de la herramienta que les permitan satisfacer sus propias necesidades. A pesar de la heterogeneidad sobre lo que los participantes quieren y cuándo lo quieren, y teniendo en cuenta que pueden ver las intervenciones digitales como irrelevantes para suplir sus necesidades individuales, este tipo de intervenciones podría ofrecer vías personalizadas. Para conseguirlo, es imprescindible que las intervenciones online dispongan de los recursos adecuados y sigan una serie de directrices en su diseño como son los Principios para el Desarrollo Digital. (Dodd et al., 2017). A pesar de estos hallazgos alentadores, deben tenerse en cuenta ciertas limitaciones a la hora de emplear las aplicaciones digitales, basadas en la web o en dispositivos móviles, no solo de cara a los pacientes, sino también de cara a los terapeutas. El despliegue de nuevas tecnologías de salud, de forma habitual, en entornos sanitarios implica desafíos adicionales que deben superarse. Esto incluye la asignación de la tecnología en el flujo de trabajo clínico diario, a la vez que son sensibles a la inversión de tiempo necesaria por parte de los diseñadores y desarrolladores web para participar plenamente en el proceso de diseño, educar a los terapeutas y pacientes, y desarrollar sistemas de soporte robustos. Además, también debe aceptarse que no todos los terapeutas o pacientes adoptarán este tipo de herramientas digitales, aunque estén bien diseñadas e integradas en los procesos de atención sanitaria, requiriendo una mayor comprensión de las limitaciones de las herramientas digitales en Salud Mental (Bauer et al., 2018). 
Principios de Diseño en las aplicaciones de m-Salud Mental orientadas al control psicosocial

\section{Agradecimientos}

Este articulo ha sido desarrollado gracias al estudio y análisis de casos realizados con motivo de la tesis doctoral "Evaluación de la Experiencia de Usuario (UX) mediante la aplicación interactiva e-Terapia orientada a la prevención de recaídas y al control de la sintomatología en personas con Trastorno Bipolar".

\section{Referencias}

Arnberg, FK., Linton, SJ., Hultcrantz, M., Heintz, E., Jonsson, U. (2014). Internet-delivered psychological treatments for mood and anxiety disorders: a systematic review of their efficacy, safety, and costeffectiveness. PLoS One;9:e98118. DOI: 10.1371/jounal.pone.0098118

Bauer, AM., Hodsdon, S., Bechtel, JM., Fortney, CN. (2018). Applying the Principles for Digital Development: Case Study of a Smartphone App to Support Collaborative Care for Rural Patients with Posttraumatic Stress Disorder or Bipolar Disorder. Journal of Medical Internet Research; 20(6): e10048. DOI: 10.2196/10048:10.2196/10048.

Dodd, A., Mallinson, S., Griffiths, M., Morris, R., Jones, SH., Lobban, F. (2017). Users' experiences of an online intervention for bipolar disorder: important lessons for design and evaluation. Evidence-Based Mental Health. Vol 20 No 4; pp. 133-139.

Hidalgo-Mazzei, D., Mateu, A., Reinares, M. (2015). Internet-based psychological interventions for bipolar disorder: review of the present and insights into the future. Journal of Affective Disorder; 188; pp. 1-13. DOI: 10.1016/j.jad.2015.08.005

Murnane, EL., Cosley, D., Chang, P., Guhal, S., Frank, E., Gay, G., Matthews, M., (2016) Self-monitoring practices, attitudes, and needs of individuals with bipolar disorder: implications for the design of technologies to manage mental health. Journal of the American Medical Informatics Association; 23: pp. 477-484. DOI:10.1093/jamia/ocv165.

Naslund, JA., Marsch, LA., McHugo, GJ., Bartels, SJ. (2015). Emerging mHealth and eHealth interventions for serious mental illness: a review of the literature. Journal of Mental Health; 24; pp. 321-32. DOI: 10.3109/09638237.2015.1019054

Waugaman A. (2016). UNICEF Innovation. Washington, DC: The Principles for Digital Development Working Group. From principle to practice: implementing the principles for digital development. En http://www.unicefstories.org/wp-content/uploads/2013/08/From_Principle_to_Practice.pdf Consultado el 30 de marzo de 2019. 